\title{
Ring chromosome 13: lack of distinct syndromes based on different breakpoints on 13q
}

\author{
C A Brandt, J M Hertz, M B Petersen, F Vogel, H Noer, M Mikkelsen
}

\begin{abstract}
A stillborn male child with anencephaly and multiple malformations was found to have the karyotype $46, X Y, r(13)$ (p11q21.1). The breakpoint at 13q21.1, determined by high resolution banding, is the most proximal breakpoint ever reported in patients with ring chromosome 13. In situ hybridisation with the probe L1.26 confirmed the derivation from chromosome 13 and DNA polymorphism analysis showed maternal origin of the ring chromosome.

Our results, together with a review of previous reports of cases with ring chromosome 13 with identified breakpoints, could neither support the theory of distinct clinical syndromes based on different breakpoints on 13q nor correlate the severity of symptoms with instability of the ring.

( 7 Med Genet 1992;29:704-8)
\end{abstract}

A ring chromosome formation of an acrocentric chromosome is often associated with increased severity of clinical symptoms compared to deletion of the same segment but without ring formation. ${ }^{1}$ The most consistent clinical symptoms have been reported in ring chromosome 13 patients. ${ }^{1}$ Since the first report of a ring chromosome $13,{ }^{2}$ many attempts have been made to correlate the clinical phenotype to cytogenetic findings. ${ }^{134}$

Niebuhr and Ottosen ${ }^{4}$ identified three groups based on the clinical findings among patients with ring chromosome 13 and interstitial deletions in 13q. Breakpoints at $13 \mathrm{q} 33$ or
$13 q 34$ are found in the majority of reported cases of ring chromosome 13. A smaller group shows breakpoints at $13 \mathrm{q} 31$ or $13 \mathrm{q} 32$; more proximal breakpoints in the long arm have only been reported twice ${ }^{56}$ and one was derived from a maternal Robertsonian translocation chromosome ( $t(13 q 13 q)){ }^{6}$

We present a case of ring chromosome 13 with very severe symptoms, namely anencephaly and multiple malformations. Anencephaly has only been reported in one previous case by Schmidt et al. ${ }^{5}$ The previously reported 22 cases of ring chromosome 13 with identified breakpoints are reviewed. ${ }^{13-19}$ The clinical findings were compared to the breakpoints and the stability of the ring, in an effort to test the theory of several clinical subgroups of patients with ring chromosome $13 .{ }^{120}$

\section{Material and methods \\ CASE REPORT}

A male infant was stillborn at 29 weeks' gestation. Anencephaly was suspected after ultrasound investigation and labour was induced. He was the first child of a 25 year old mother and a 30 year old father. Both parents were healthy and non-consanguineous. The pregnancy was normal until the actual referral based on the symptoms of intrauterine growth retardation. The child was in breech position in labour which was otherwise uneventful. Birth weight was $650 \mathrm{~g}$ (less than 10th centile for 29 weeks of gestation) and birth length was $31 \mathrm{~cm}$.

Observation of the infant at birth showed (fig 1) anencephaly, cranial bone defect at the vault, broad nasal bridge, hypertelorism, low
Genetics, The
Bartholin Building,
University of Aarhus,

DK-8000 Aarhus C,

Denmark.

C A Brandt

J M Hertz

Department of

Medical Genetics, The

John F Kennedy

Institute, DK-2600

Glostrup, Denmark.

M B Petersen

M Mikkelsen

Department of Gynecology and

Obstetrics, Hospital of Aalborg, DK-9000

Aalborg, Denmark.

F Vogel

Department of

Pathology, Hospital of Aalborg, DK-9000

Aalborg, Denmark.

$\mathrm{H}$ Noer

Correspondence to

Dr Brandt.

Received 18 March 1992. Accepted 31 March 1992
Figure 1 The stillborn male infant with a ring chromosome 13. Note the proximally placed thumb on the right hand. 
set ears, high arched palate, short philtrum, micrognathia, hypoplastic thumb on the left hand, imperforate anus, and varus position of the left foot with abnormal overlapping second toe.

Pathological examination showed a small baby with a $7 \mathrm{~cm}$ long cranium boine defect at the vault. Typical anencephaly with sparse cortical tissue and no sign of cerebellar tissue was found. Hypoplasia of the gallbladder was detected at necropsy and by histological examination. The suprarenal glands seemed hypoplastic but had normal histological architecture. Both kidneys were normal but a stenotic area was found in the left ureter near the bladder. No genital malformations were found. The imperforate anal canal was covered with skin but no atresia was observed.

\section{CYTOGENETIC ANALYSIS}

Metaphase chromosome preparations from the proband were made from cultured skin fibroblasts (H 131-90) and stained with quinacrine mustard (QFQ banding) using standard methods. A total of 200 metaphases was analysed. Chromosome analyses of the parents were performed on lymphocyte cultures and both were found to have normal karyotypes. To determine the breakpoints involved in formation of the ring chromosome, high resolution banding analysis was performed using prometaphase chromosomes with RBA, GTG, and QFQ banding and AgNOR silver staining.

\section{MOLECULAR ANALYSIS}

Genomic DNA was isolated from cultured fibroblasts from the proband and from parental leucocytes. The DNA polymorphisms studied were the dinucleotide repeats of locus $D 13 S 71^{21}$ and the FLT1 gene assigned to chromosome $13 \mathrm{q} 12 .{ }^{22}$ The polymorphisms were detected after PCR amplification of genomic DNA. ${ }^{23}$ The primers used and the PCR programmes were as previously described. ${ }^{2122}$ End labelling of primers, polyacrylamide gel electrophoresis of the amplification products, and autoradiography have been described elsewhere. $^{2425}$

\section{IN SITU HYBRIDISATION}

The preparation of human metaphase spreads and the in situ hybridisation protocol were performed essentially as described by Pinkel $e t$ $a l^{26}$ with only a few modifications. The probe L1.26 (from American Type Culture Collection), specific for $\alpha$ satellite DNA sequences from the centromeres of chromosomes 13 and 21 , was used. The probe was labelled by nick translation with Biotin-11-dUTP (Sigma) according to standard methods. ${ }^{27}$ Hybridised probe was detected with fluorescein conjugated avidin and amplified using biotinylated anti-avidin antibody together with a new layer of fluorescein conjugated avidin. Chromosomes and interphase nuclei were counterstained by propidium iodide (Sigma). Slides were mounted in antifade solution and examined with a Leitz Diaplan microscope connected to a confocal laser scanning computer. Photographs were taken on an Agfachrome 50 RS colour slide film or Kodak technical pan TP651 black and white film directly from the screen.

\section{Results}

Cytogenetic analysis showed 46 chromosomes including a small ring chromosome 13 in $92 \%$ of the cells (fig 2, table 1 ). In $8 \%$ of the cells, only 45 chromosomes were seen and no ring chromosome (table 1). A normal cell line was not found in 200 cells examined. The ring chromosome was negative by silver staining indicating that no satellites were present. High resolution banding showed the breakpoints to be at $\mathrm{p} 11$ and $\mathrm{q} 21.1$, and the karyotype was thus 46,XY,r(13) (p11q21.1) (fig 2).

The results of the DNA polymorphism analysis are given in table 2. Locus FLT1 showed two alleles in the proband, while D13S71 showed only one allele with no maternal allele contribution (fig 3 ), indicating maternal origin of the ring and showing that the D13S71 locus is distal to the breakpoint of the ring chromosome (13q21.1).

By using in situ hybridisation with a centromere probe specific for chromosomes 13 and 21 (L 1.26), 68 metaphases showed four signals (table 1). In 32 metaphases only three signals were detected and in two metaphases five signals were found, indicating a dicentric ring chromosome (table 1, fig 4).

\section{Discussion}

We have reviewed 22 previous cases of ring chromosome 13 with identified breakpoints

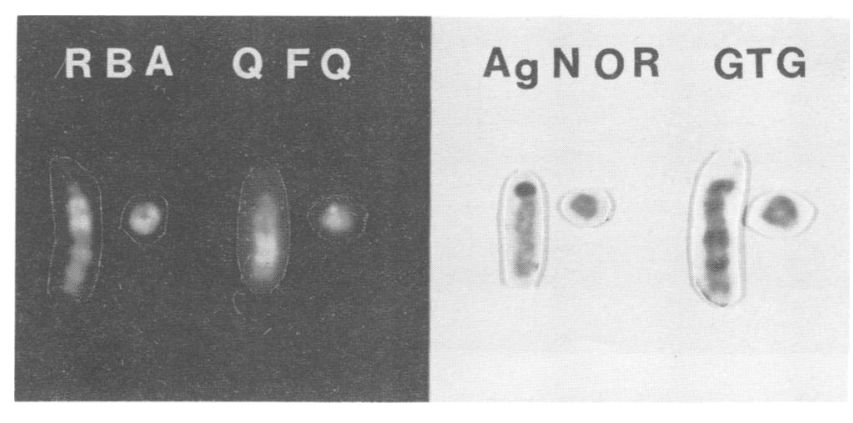

Figure 2 Chromosomes 13 in prometaphase stained with $R B A, Q F Q, A g N O R$, and $G T G$ banding techniques.
Figure 3 Autoradiogram showing the alleles of the D13S71 (CA)n polymorphism in the ring chromosome 13 family. 
Table 1 Distribution of cell lines in a case with ring chromosome 13.

\begin{tabular}{lcc}
\hline & QFQ banding & in situ hybridisation \\
\hline 46,XY,r(13) single ring & 184 & 68 \\
45,XY,-13 & 16 & 32 \\
68,XY,r(13) double ring & 0 & 2 \\
Total cell count & 200 & 102
\end{tabular}

QFQ banding and later in situ hybridisation were performed on the same fibroblast culture, which reflects the in vitro instability of the ring.

Table 2 DNA polymorphism analysis in a ring chromosome 13 family.

\begin{tabular}{lll}
\hline Locus & D13S71 (CA)n & FLT1 (GT)n \\
Father & 13 & 12 \\
r(13) & 1 & 12 \\
Mother & 24 & 22 \\
\hline
\end{tabular}

The numbers represent the different alleles at a specific locus.

(tables 3 and 4). All cases had microcephaly/ anencephaly, severe mental retardation, and multiple malformations. Other clinical findings, found in more than $50 \%$ of cases, include: growth retardation (21/23), facial dysmorphism (for example, epicanthus, broad nasal bridge, and hypertelorism) $(22 / 23)$, abnormal ears (14/23), high arched palate (14/ 23 ), and foot or toe abnormalities (12/23). Clinical traits, found in more than $25 \%$ of the cases but less than $50 \%$, are low hair line or alopecia, micrognathia, short philtrum, short or webbed neck, functional renal defect, and genital, anal, skeletal, and thumb anomalies. Less frequent abnormalities associated with ring chromosome 13 were hypoplasia of different organs, cardiac anomalies, epilepsy and pigment anomalies.

Of 23 cases of ring chromosome 13 with identified breakpoints the mean gestational age at birth was 37.8 weeks and the mean birth weight was $2027 \mathrm{~g}$, which is below the 10 th centile for 38 weeks. The sex distribution was equal, 11 were males and 12 were females. The parental origin of the ring chromosome has only been determined in seven out of 23 cases (tables 3 and 4).

According to the classification of Niebuhr and Ottosen ${ }^{4}$ and Niebuhr, ${ }^{20}$ based on specific

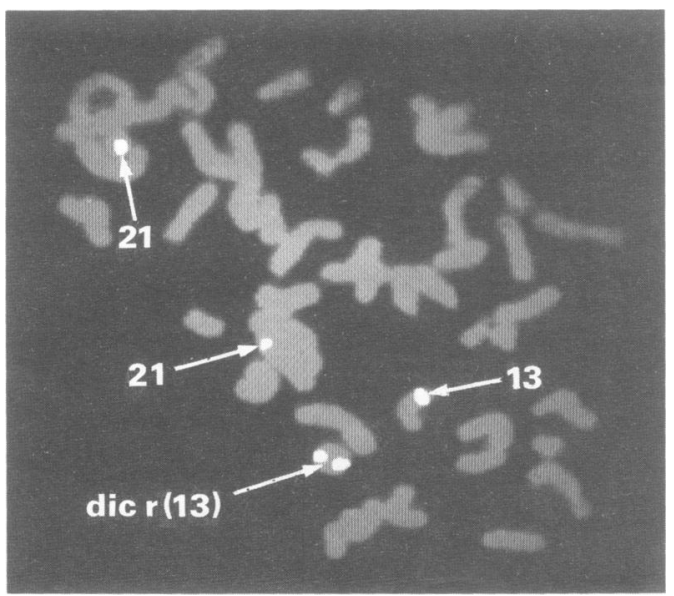

Figure 4 The result of in situ hybridisation on metaphase spread using probe L1.26 specific for chromosomes 13 and 21. Two signals in close proximity indicate a double ring chromosome 13. clinical features among patients with ring chromosome 13 or deletion of the long arm of chromosome 13, it was suggested that breakpoints at $13 \mathrm{q} 33$ and $13 \mathrm{q} 34$ are frequently found in patients with severe mental retardation, microcephaly with true hypertelorism, frontal bossing erasing the nasal bridge, protruding upper incisors, and large ears with deep sulci. In the second group, they recorded breakpoints at 13q31 and 13q32. These patients exhibited almost all the features of the first group in addition to aplasia or hypoplasia of the thumbs, foot or toe abnormalities, severe genital malformations, anal atresia, and eye malformations. The third group, with a breakpoint at $13 \mathrm{q} 21$, was characterised by a large number of patients with retinoblastoma. Niebuhr and Ottosen ${ }^{4}$ found no cases with a ring chromosome 13 in the latter group, but only patients with interstitial deletion in the $13 q 21$ region. These probably had deletion of $13 q 14$, because more recent reviews ${ }^{28}$ have shown that a candidate gene for retinoblastoma lies within the $13 \mathrm{q} 14$ region.

We have reviewed 22 previous cases of ring chromosome 13 with identified breakpoints on $13 \mathrm{q}$ and ordered them into three groups according to the site of the breakpoint. The clinical features are shown in tables 3 and 4 in order of increasing size of the deleted segment. According to tables 3 and 4, facial dysmorphism is more often found in cases with larger deletions, that is, in groups 2 and 3 rather than in group 1, and so are malformations of the genital and anal region and of the extremities, but not exclusively so. Aplasia/hypoplasia or proximally placed thumbs are found with equal frequency in the three groups. Even within the three groups, where the breakpoints are quite similar, considerable phenotypic variability is found. However, the most striking difference between the groups is that in group 3 two out of three cases have anencephaly. There was no information about microcephaly in the third case, ${ }^{6}$ but it was a very small fetus which was stillborn at term. Thus, a distinct clinical syndrome for each group based on different breakpoints on $13 \mathrm{q}$ seems unlikely. An attempt to map different clinical traits to specific segments on 13q using ring chromosome 13 patients will therefore be inaccurate.

It has been a subject of discussion whether the stability of the ring could have any effect on the severity of symptoms. ${ }^{71013} \mathrm{~A}$ stable ring has only been reported twice, ${ }^{1617}$ but the total cell count in those reports was only 36 and 50 , respectively. Thus, at least 21 cases out of 23 seem to have a considerable degree of instability showing as loss of the ring or the presence of a single, double, or triple ring. Ascertainment was made from different tissue cultures, for example, blood, skin, and amniotic fluid (tables 3 and 4), so a comparison of the influence of mitotic instability on the clinical severity should be made with caution. Nevertheless, the present review shows that instability among ring chromosomes 13 does not influence the severity of symptoms. This conclusion is also in agreement with the findings of McCorquodale et al. ${ }^{13}$ 
Table 3 Ring chromosomes 13 with breakpoints at $q 34 / q 33$.

\begin{tabular}{|c|c|c|c|c|c|c|c|c|c|c|c|c|c|c|c|}
\hline & \multicolumn{14}{|c|}{ Reference } & \multirow[b]{2}{*}{$\begin{array}{l}\text { All } 14 \text { cases } \\
\text { (mean value) }\end{array}$} \\
\hline & 7 & 3 & 8 & 9 & 10 & $\begin{array}{c}11 \\
\text { Case } \\
2\end{array}$ & $\begin{array}{c}12 \\
\text { Case } \\
2\end{array}$ & 13 & 14 & 15 & 16 & $\begin{array}{c}17 \\
\text { Case } \\
1\end{array}$ & $\begin{array}{c}11 \\
\text { Case }\end{array}$ & $\begin{array}{c}1 \\
\text { Case } \\
3\end{array}$ & \\
\hline $\begin{array}{l}\text { Gestational age (wk) } \\
\text { Birth weight }(\mathrm{g}) \\
\text { Birth length }(\mathrm{cm}) \\
\text { Sex } \\
\text { Age mother/father }\end{array}$ & $\begin{array}{c}\mathrm{NI} \\
2050 \\
48 \\
\mathrm{M} \\
31 / 31\end{array}$ & $\begin{array}{c}40 \\
2700 \\
\text { NI } \\
\text { M } \\
\text { NI }\end{array}$ & $\begin{array}{c}41 \\
2350 \\
47 \\
M \\
23 / 26\end{array}$ & $\begin{array}{c}38 \\
2160 \\
\mathrm{NI} \\
\mathrm{F} \\
41 / 48\end{array}$ & $\begin{array}{c}40 \\
\text { NI } \\
\text { NI } \\
\text { F } \\
21 / 23\end{array}$ & $\begin{array}{c}<37 \\
1600 \\
40 \\
\mathrm{~F} \\
\mathrm{NI}\end{array}$ & $\begin{array}{c}\mathrm{NI} \\
2350 \\
48 \\
\mathrm{M} \\
20 / 23\end{array}$ & $\begin{array}{c}37 \\
2155 \\
46 \\
\mathrm{~F} \\
17 / \mathrm{NI}\end{array}$ & $\begin{array}{c}40 \\
1950 \\
48 \\
M \\
21 / \mathrm{NI}\end{array}$ & $\begin{array}{c}\text { NI } \\
\text { NI } \\
\text { NI } \\
\text { F } \\
25 / 23\end{array}$ & $\begin{array}{c}36 \\
2100 \\
\mathrm{NI} \\
\mathrm{F} \\
33 / 36\end{array}$ & $\begin{array}{c}40 \\
2660 \\
47 \\
M \\
\text { NI }\end{array}$ & $\begin{array}{c}\text { NI } \\
\text { NI } \\
\text { NI } \\
\text { F } \\
41 / 46\end{array}$ & $\begin{array}{c}44 \\
4350 \\
\text { NI } \\
\text { F } \\
17 / 19\end{array}$ & $\begin{array}{c}39 \cdot 3 \\
2402 \\
46 \cdot 3 \\
6 / 8 \\
26.4 / 30.6\end{array}$ \\
\hline $\begin{array}{l}\text { Anencephaly } \\
\text { Microcephaly } \\
\text { Mental retardation } \\
\text { Growth retardation } \\
\text { Hypotonia } \\
\text { Facial dysmorphism } \\
\text { Protruding forehead } \\
\text { Hair (low line, alopecia) } \\
\text { Broad nasal bridge } \\
\text { Epicanthus } \\
\text { Hypertelorism } \\
\text { Micrognathia } \\
\text { Short philtrum } \\
\text { High arched palate } \\
\text { Abnormal ears } \\
\text { Low set ears } \\
\text { Short neck/webbing } \\
\text { Other abnormalities }\end{array}$ & $\begin{array}{l}+ \\
+ \\
+ \\
+ \\
+\end{array}$ & $\begin{array}{l}+ \\
+ \\
+ \\
+ \\
\text { L } \\
+ \\
+\end{array}$ & $\begin{array}{l}+ \\
- \\
-\end{array}$ & $\begin{array}{l}+ \\
+ \\
+ \\
+ \\
\text { Al } \\
+ \\
- \\
- \\
- \\
+ \\
- \\
- \\
\mathrm{Pi}\end{array}$ & $\begin{array}{l}+ \\
\pm \\
+ \\
+ \\
+1- \\
\text { Ho }\end{array}$ & $\begin{array}{l}+ \\
+ \\
+\end{array}$ & $\begin{array}{l}+ \\
+ \\
+ \\
+\end{array}$ & $\begin{array}{l}+ \\
+ \\
+ \\
+ \\
+ \\
+ \\
+ \\
+ \\
+\end{array}$ & $\begin{array}{l}+ \\
+ \\
+ \\
+\end{array}$ & $\begin{array}{c}+ \\
+ \\
+ \\
-1+\end{array}$ & $\begin{array}{l}+ \\
+ \\
+ \\
+ \\
+ \\
+ \\
+ \\
+ \\
+ \\
+\end{array}$ & $\begin{array}{l}+ \\
+ \\
+\end{array}$ & $\begin{array}{c}+ \\
- \\
\mathrm{Cl}+\end{array}$ & $\begin{array}{l}+ \\
+ \\
+ \\
+\end{array}$ & $\begin{array}{l}0 \%(0 / 14) \\
100 \%(14 / 14) \\
100 \%(14 / 14) \\
86 \%(12 / 14) \\
7 \%(1 / 14) \\
93 \%(13 / 14) \\
21 \%(3 / 14) \\
33 \%(5 / 14) \\
64 \%(9 / 14) \\
64 \%(9 / 14) \\
43 \% \%(6 / 14) \\
29 \%(4 / 14) \\
14 \%(2 / 14) \\
43 \%(6 / 14) \\
50 \%(7 / 14) \\
21 \%(3 / 14) \\
29 \%(4 / 14)\end{array}$ \\
\hline $\begin{array}{l}\text { Renal defect (functional) } \\
\text { Genital malformation } \\
\text { Imperforate anus } \\
\text { Skeletal anomalies } \\
\text { Foot anomalies } \\
\text { Toe anomalies } \\
\text { Hand anomalies } \\
\text { Hypoplasia 1st finger }\end{array}$ & $\begin{array}{l} \pm \\
= \\
-\end{array}$ & - & $\begin{array}{l}- \\
- \\
- \\
+\end{array}$ & - & $\begin{array}{l}+ \\
+ \\
+ \\
+\end{array}$ & + & $\begin{array}{l}- \\
+ \\
-\end{array}$ & - & + & $\begin{array}{l}+ \\
+ \\
+ \\
+ \\
+ \\
+ \\
\mathrm{Pp}\end{array}$ & $\begin{array}{c}\overline{+} \\
+ \\
P p\end{array}$ & $\begin{array}{l}- \\
\overline{-} \\
- \\
-\end{array}$ & $\begin{array}{l}- \\
+ \\
+ \\
+ \\
+ \\
+ \\
\mathrm{Pp}\end{array}$ & $\begin{array}{l}+ \\
+\end{array}$ & 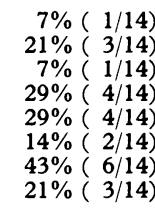 \\
\hline $\begin{array}{l}\text { Cytogenetic studies } \\
\text { Cultures ( } \mathrm{S} / \mathbf{B} / \mathbf{M} / \mathrm{A}) \\
\text { Origin of ring (mother/father) } \\
\text { Total cell count } \\
45,-\mathrm{r}(13)(\%) \\
46, \mathrm{r}(13)(\%) \\
46, \mathrm{r}(13) \text { double ring }(\%) \\
\text { Breakpoints }\end{array}$ & $\begin{array}{c}\text { S/B } \\
\text { NI } \\
600 \\
6 \\
84 \\
7 \\
\text { p11q34 }\end{array}$ & $\begin{array}{l}\text { B } \\
\text { NI } \\
\text { NI } \\
\text { US } \\
\text { q34 }\end{array}$ & $\begin{array}{c}\text { NI } \\
\text { NI } \\
\text { NI } \\
0 \\
95 \\
5 \\
\text { p13q34 }\end{array}$ & $\begin{array}{c}\text { S/B } \\
\text { NI } \\
100 \\
5 \\
92 \\
3 \\
\text { p11q34 }\end{array}$ & $\begin{array}{c}\text { B } \\
\mathrm{NI} \\
228 \\
5 \\
80 \\
12 \\
\text { p11q34 }\end{array}$ & $\begin{array}{c}\text { B } \\
\text { NI } \\
351 \\
8 \\
82 \\
5 \\
\text { p12q34* }\end{array}$ & $\begin{array}{c}\mathbf{B} \\
\mathbf{M} \\
\mathrm{NI} \\
\mathrm{US}\end{array}$ & $\begin{array}{c}\text { S/B } \\
M \\
500 \\
0.2 \% \\
96 \\
4 \\
\text { p13q34 }\end{array}$ & $\begin{array}{c}\mathrm{S} / \mathrm{B} \\
\mathrm{M}+ \\
294 \\
17 \\
81 \\
2 \\
\mathrm{p} 12 \mathrm{q} 33\end{array}$ & $\begin{array}{c}\text { S/B } \\
\text { F } \\
500 \\
\text { US } \\
70 \\
\text { p11q33 }\end{array}$ & $\begin{array}{c}\text { B } \\
\text { NI } \\
36 \\
0 \\
100 \\
0 \\
\text { p12q33 }\end{array}$ & $\begin{array}{c}\text { B } \\
\text { NI } \\
50 \\
0 \\
100 \\
0 \\
\mathrm{q}^{3} 3^{*}\end{array}$ & $\begin{array}{c}\text { B } \\
\text { NI } \\
309 \\
7 \\
85 \\
4 \\
\text { p11933* }\end{array}$ & $\begin{array}{c}\text { B/M } \\
\text { NI } \\
158 \\
2 \\
89 \\
5 \\
\text { p1lq33 }\end{array}$ & \\
\hline
\end{tabular}

$\mathrm{Aa}=$ anteriorly placed anus; $\mathrm{Al}=$ alopecia areata; cultures: skin/blood/bone marrow/amniotic fluid; $\mathrm{Ca}=$ cardiac anomaly; $\mathrm{Cl}=$ cleft lip; $\mathrm{Co}=$ coloboma; Ep $=$ epilepsy $\mathrm{Ho}=$ hypoplasia of different organs; NI = no information; $\mathrm{Pi}=$ pigment anomaly; $\mathrm{Pp}=$ proximally placed; US = unstable; ${ }^{*}$ prometaphase technique; tmaterna translocated chromosome.

Table 4 Ring chromosomes 13 with breakpoints at q32/q31 (left 6 cases) and q2/q1 (right 3 cases).

\begin{tabular}{|c|c|c|c|c|c|c|c|c|c|c|c|c|}
\hline & \multicolumn{6}{|c|}{ References } & \multirow[b]{2}{*}{$\begin{array}{l}\text { All } 6 \text { cases } \\
\text { (mean value) }\end{array}$} & \multicolumn{3}{|c|}{ References } & \multirow[b]{2}{*}{$\begin{array}{l}\text { All } 3 \text { cases } \\
\text { (mean value) }\end{array}$} & \multirow[b]{2}{*}{$\begin{array}{l}\text { All } 23 \text { cases } \\
\text { (mean value) }\end{array}$} \\
\hline & 18 & $\begin{array}{c}12 \\
\text { Case } \\
1\end{array}$ & $\begin{array}{c}1 \\
\text { Case } \\
1\end{array}$ & $\begin{array}{c}1 \\
\text { Case } \\
2\end{array}$ & 4 & 19 & & 5 & Present & 6 & & \\
\hline $\begin{array}{l}\text { Gestational age (wk) } \\
\text { Birth weight }(\mathbf{g}) \\
\text { Birth length }(\mathrm{cm}) \\
\text { Sex } \\
\text { Age mother/father }\end{array}$ & $\begin{array}{c}\mathrm{NI} \\
2425 \\
\mathrm{NI} \\
\mathrm{M} \\
25 / 24\end{array}$ & $\begin{array}{c}\mathrm{NI} \\
1850 \\
44 \\
\mathrm{M} \\
33 / 30\end{array}$ & $\begin{array}{c}37 \\
2220 \\
\mathrm{NI} \\
\mathrm{F} \\
27 / 29\end{array}$ & $\begin{array}{c}34 \\
1680 \\
41 \\
F \\
24 / 23\end{array}$ & $\begin{array}{c}39 \\
1800 \\
42 \\
M \\
24 / 24\end{array}$ & $\begin{array}{l}\text { NI } \\
1900 \\
46 \\
M \\
27 / 31\end{array}$ & $\begin{array}{c}36 \cdot 7 \\
1979 \\
43 \cdot 3 \\
4 / 2 \\
26.7 / 26.8\end{array}$ & $\begin{array}{c}33 \\
900 \\
32 \\
\mathrm{~F} \\
41 / \mathrm{NI}\end{array}$ & $\begin{array}{c}29 \\
650 \\
31 \\
M \\
25 / 30\end{array}$ & $\begin{array}{c}40 \\
690 \\
\mathrm{NI} \\
\mathrm{F} \\
17 / \mathrm{NI}\end{array}$ & $\begin{array}{c}34 \\
747 \\
31 \cdot 5 \\
1 / 2 \\
27.7\end{array}$ & $\begin{array}{c}37 \cdot 8 \\
2027 \\
40 \cdot 2 \\
11 / 12 \\
26.7 / 29.1\end{array}$ \\
\hline $\begin{array}{l}\text { Anencephaly } \\
\text { Microcephaly } \\
\text { Mental retardation } \\
\text { Growth retardation } \\
\text { Hypotonia } \\
\text { Facial dysmorphism } \\
\text { Protruding forehead } \\
\text { Hair (low line, alopecia) } \\
\text { Broad nasal bridge } \\
\text { Epicanthus } \\
\text { Hypertelorism } \\
\text { Micrognathia } \\
\text { Short philtrum } \\
\text { High arched palate } \\
\text { Abnormal ears } \\
\text { Low set ears } \\
\text { Short neck/webbing } \\
\text { Other abnormalities }\end{array}$ & $\begin{array}{l}+ \\
+ \\
+ \\
+ \\
+ \\
+\end{array}$ & $\begin{array}{l}+ \\
+ \\
+ \\
+ \\
+ \\
\\
\text { Al } \\
+ \\
+ \\
+\end{array}$ & $\begin{array}{c}+ \\
+ \\
+ \\
+ \\
\\
+ \\
+ \\
+ \\
+ \\
+ \\
+ \\
+ \\
+ \\
\text { Co/Ho }\end{array}$ & $\begin{array}{c}+ \\
+ \\
+ \\
+ \\
+ \\
+ \\
+ \\
-1- \\
\mathrm{Ca} / \mathrm{Ho}\end{array}$ & $\begin{array}{l}+ \\
+ \\
+ \\
- \\
+ \\
+ \\
+ \\
+\end{array}$ & $\begin{array}{c}+ \\
+ \\
+ \\
+ \\
+ \\
+ \\
+ \\
+ \\
+ \\
+ \\
+ \\
+ \\
+ \\
+1+ \\
\text { Co/Ho }\end{array}$ & $\begin{array}{r}100 \%(6 / 6) \\
100 \%(6 / 6) \\
100 \%(6 / 6) \\
33 \%(2 / 6) \\
100 \%(6 / 6) \\
17 \%(1 / 6) \\
33 \%(2 / 6) \\
100 \%(6 / 6) \\
33 \%(2 / 6) \\
83 \%(5 / 6) \\
50 \%(3 / 6) \\
83 \%(5 / 6) \\
83 \%(5 / 6) \\
100 \%(5 / 6) \\
83 \%(5 / 6) \\
33 \%(2 / 6)\end{array}$ & $\begin{array}{l}+ \\
+ \\
+ \\
+ \\
+ \\
+ \\
\text { Ho }\end{array}$ & $\begin{array}{l}+ \\
\text { SB } \\
+ \\
+\end{array}$ & $\begin{array}{c}\text { NI } \\
\text { SB } \\
+ \\
+ \\
\\
+ \\
+ \\
+ \\
+ \\
+ \\
+ \\
+ \\
+ \\
\mathrm{Ca} / \mathrm{Ho}\end{array}$ & $\begin{array}{r}67 \%(2 / 3) \\
33 \%(1 / 3) \\
\\
100 \%(3 / 3) \\
0 \%(0 / 3) \\
100 \%(3 / 3) \\
0 \%(0 / 3) \\
0 \%(0 / 3) \\
100 \%(3 / 3) \\
67 \%(2 / 3) \\
100 \%(3 / 3) \\
33 \%(1 / 3) \\
100 \%(3 / 3) \\
100 \%(3 / 3) \\
67 \%(2 / 3) \\
67 \%(2 / 3) \\
33 \%(1 / 3)\end{array}$ & $\begin{array}{r}9 \%(2 / 23) \\
91 \%(21 / 23) \\
100 \%(20 / 20) \\
91 \%(21 / 23) \\
13 \%(3 / 23) \\
96 \%(22 / 23) \\
17 \%(4 / 23) \\
30 \%(7 / 23) \\
78 \%(18 / 23) \\
57 \%(13 / 23) \\
61 \%(14 / 23) \\
35 \%(8 / 23) \\
43 \%(10 / 23) \\
61 \%(14 / 23) \\
61 \%(14 / 23) \\
43 \%(10 / 23) \\
30 \%(7 / 23)\end{array}$ \\
\hline $\begin{array}{l}\text { Renal defect (functional) } \\
\text { Genital malformation } \\
\text { Imperforate anus } \\
\text { Skeletal anomalies } \\
\text { Foot anomalies } \\
\text { Toe anomalies } \\
\text { Hand anomalies } \\
\text { Hypoplasia 1st finger }\end{array}$ & $\begin{array}{l}+ \\
+\end{array}$ & $\overline{+}$ & $\begin{array}{l}+ \\
+ \\
\text { Aa } \\
+ \\
+ \\
+ \\
+\end{array}$ & $\begin{array}{l}+ \\
+ \\
+ \text { Aa } \\
+ \\
+ \\
+ \\
+\end{array}$ & $\begin{array}{l}+ \\
+ \\
+ \\
+ \\
+ \\
\text { A }\end{array}$ & $\begin{array}{l}+ \\
+ \\
+ \\
+ \\
+ \\
+ \\
+ \\
+\mathrm{Pp}\end{array}$ & $\begin{aligned} 33 \%(2 / 6) \\
100 \%(6 / 6) \\
50 \%(3 / 6) \\
67 \%(4 / 6) \\
50 \%(3 / 6) \\
50 \%(3 / 6) \\
83 \%(5 / 6) \\
33 \%(2 / 6)\end{aligned}$ & $\begin{array}{l}+ \\
+ \\
+ \\
+ \\
+ \\
+ \\
+ \\
+\end{array}$ & $\begin{array}{l}- \\
+ \\
+ \\
+ \\
+\end{array}$ & $\begin{array}{c}+ \\
+ \\
\mathbf{A a}\end{array}$ & $\begin{array}{r}67 \%(2 / 3) \\
67 \%(2 / 3) \\
100 \%(3 / 3) \\
33 \%(1 / 3) \\
67 \%(2 / 3) \\
100 \%(3 / 3) \\
67 \%(2 / 3) \\
67 \%(2 / 3)\end{array}$ & $\begin{array}{l}22 \%(5 / 20) \\
48 \%(11 / 23) \\
30 \%(7 / 23) \\
39 \%(9 / 23) \\
39 \%(9 / 23) \\
35 \%(8 / 23) \\
57 \%(13 / 23) \\
30 \%(7 / 23)\end{array}$ \\
\hline $\begin{array}{l}\text { Cytogenetic studies } \\
\text { Cultures }(\mathrm{S} / \mathrm{B} / \mathrm{M} / \mathrm{A}) \\
\text { Origin of ring (mother/father) } \\
\text { Total cell count } \\
46,-\mathrm{r}(13)(\%) \\
46, \mathrm{r}(13)(\%) \\
46, \mathrm{r}(13) \text { double ring }(\%) \\
\text { Breakpoints }\end{array}$ & $\begin{array}{c}\text { S/B } \\
\\
144 \\
3 \\
94 \\
3 \\
\text { q32 }\end{array}$ & $\begin{array}{c}\text { S/B } \\
\text { F } \\
\text { NI } \\
\text { US } \\
\text { p13q32 }\end{array}$ & $\begin{array}{c}\text { B } \\
\text { NI } \\
58 \\
3 \\
88 \\
3 \\
\text { p11q32 }\end{array}$ & $\begin{array}{c}\text { B } \\
\text { NI } \\
126 \\
5 \\
79 \\
2 \\
\text { p11q32 }\end{array}$ & $\begin{array}{c}\text { S/B } \\
\text { NI } \\
294 \\
12 \\
79 \\
2 \\
\text { p12q31 }\end{array}$ & $\begin{array}{c}\text { B } \\
\text { NI } \\
\text { NI }\end{array}$ & & $\begin{array}{c}\text { A } \\
\text { NI } \\
30 \\
7 \\
93 \\
\\
\text { q2? }\end{array}$ & $\begin{array}{c}S \\
M \\
200 \\
8 \\
92 \\
\\
\text { p11q21.1* }\end{array}$ & $\begin{array}{c}\mathrm{S} \dagger \\
\mathrm{M} \\
\mathrm{NI} \\
9 \\
\\
\text { q11q14* }\end{array}$ & & $5 / 2$ \\
\hline
\end{tabular}

$\mathrm{A}=$ aplasia; $\mathrm{Aa}=$ anteriorly placed anus; $\mathrm{Al}=$ alopecia areata; cultures slin/blood/bone marrow/amniotic fluid; $\mathrm{Ca}=\mathrm{cardiac}$ anomaly; $\mathrm{Co}=$ coloboma; $\mathrm{Ha}=\mathrm{hypopla}-$ sia of the adrenals; $\mathrm{Ho}=$ hypoplasia of different organs; $\mathrm{Mo}=$ micrcphthalmia; $\mathrm{NI}=$ no information; $\mathrm{P}=$ pigment anomaly; $\mathrm{Pp}=\mathrm{proximally}$ placed; $\mathrm{SB}=\mathrm{stillborn}$ US = unstable; * prometaphase technique; tparental translocation chromosome. 
Reviewing the previously reported cases of ring chromosome 13, we could find no support for the existence of different syndromes based on different breakpoints on 13q or instability of the ring. However, this report shows that larger deletions of $13 q$ are more likely to cause a severe clinical outcome than smaller deletions and that deletion of the segment $13 q 2 \rightarrow$ qter in ring chromosome 13 can reflect the most serious symptom ever reported among patients with ring chromosomes, namely anencephaly.

We would like to thank Helle $\mathrm{H}$ Strømkjær, Anni Hallberg, and Connie Lund for skilful technical assistance. This work was supported by grant 5.18.10.03 from the Danish Human Genome Research Programme (CB, MBP, MM).

1 Martin NJ, Harvey PJ, Pearn JH. The ring chromosome 13 syndrome. Hum Genet 1982;61:18-23.

2 Lejeune J, Lafourcade J, Berger R, et al. Le phénotype [Dr] étude de trois cas de chromosomes $\mathrm{D}$ en anneau. An etude de trois cas de chrom
Genet (Paris) 1968;11:79-87.

3 Noel B, Quack B, Rethoré MO. Partial deletion and trisomies of chromosome 13; mapping of bands associated with particular malformations. Clin Genet 1976;9:593 602 .

4 Niebuhr E, Ottosen J. Ring chromosome $\mathrm{D}(13)$ associated with multiple congenital malformations. Ann Gene (Paris) 1973;16:157-66

5 Schmid W, Mühlethaler JP, Briner J, Knechtli H. Ring chromosome 13 in a polymalformed anencephalic. Humangenetik 1975;27:63-6.

6 Stetten G, Tuck-Muller CM, Blakemore KJ, et al. Evidence for involvement of a Robertsonian translocation 13 chromosome in formation of a ring chromosome 13. Mol Biol Med 1990;7:479-84.

7 Hoo JJ, Obermann U, Cramer H. The behavior of ring chromosome 13. Humangenetik 1974;24:161-71.

8 Lowry RB, Dill FJ. C-ring 13 chromosome associated with microcephaly, congenital heart defect, intrauterine growth retardation, and abnormal skin pigmentation. Birth Defects 1977;13:216-21.

9 Verma RS, Dosik, H, Chowdhry IH, Jhaveri RC. Ring chromosome 13 in a child with minor dysmorphic features. Am f Dis Child 1978;132:1018-21.

10 Hernandez A, Garcia-Cruz D, Plascencia L, et al. Some clinical and cytogenetic observations on a ring chromosome 13 (p1lq34). Ann Genet (Paris) 1979;22:221-4.

11 Jones IM, Palmer CG, Weaver DD, Hodes ME. Study of two cases of ring 13 chromosome using high-resolution banding. Am $\mathcal{F}$ Hum Genet 1981;33:252-61.

12 Steinbach P, Drews K, Horstmann W, Barbi G, Scholz W Two cases of ring chromosome 13. Chromosome banding patterns and mosaic configuration. Ann Genet (Paris) 1981;24:152-7.

13 McCorquodale MM, Kolacki P, Kurczynski TW, Baugh, E. Tissue-specific mosaicism for the stability of a ring 13 chromosome. ₹ Ment Defic Res 1986;30:389-99.

14 Niebuhr E. Reexamination of a family with a $t(13 q 14 q)$ and a ring D(13) child. Ann Genet (Paris) 1973;16:199-202.

15 Magenis RE, Wyandt HE, Overton KM, Macfarlane J. Parental origin of a ring 13 chromosome in a female with Parental origin of a ring 13 chromosome in a femal
multiple anomalies. Hum Genet 1976;33:181-6.

16 Cossu P, Diana G, Mameli M, et al. A case of D13 ring chromosome. Hum Genet 1979;46:111-4.

17 Lagergren M, Börjeson M, Mitelman F. Prophase analysis of ring chromosome 13 - an attempt at phenotypekaryotype correlation. Hereditas 1980;93:231-3.

18 Fryns JP, Deroover J, Van den Berghe H. Malformative syndrome with ring chromosome 13. Humangenetik 1974;24:235-40.

19 Parcheta B, Wisniewski L, Piontek E, et al. Clinical features in a case with ring chromosome 13. Eur 7 Pediat 1985;144:409-12.

20 Niebuhr E. Partial trisomies and deletions of chromosome 13. In: Yunis JJ, ed. New chromosomal syndromes. New York: Academic Press, 1977:273-99.

21 Weber JL, Kwitek AE, May PE. Dinucleotide repeat polymorphism at the D13S71 locus. Nucleic Acids Res morphism at

22 Polymeropoulos MH, Rath DS, Xiao H, Merril CR. Dinucleotide repeat polymorphism at the human fmsrelated tyrosine kinase gene (FLT1). Nucleic Acids Res 1990;19:2803.

23 Saiki RK, Gelfand DH, Stoffel S, et al. Primer-directed enzymatic amplification of DNA with a thermostable DNA polymerase. Science 1988;239:487-91.

24 Economou EP, Bergen AW, Warren AC, Antonarakis SE. The polydeoxyadenylate tract of Alu repetitive elements is polymorphic in the human genome. Proc Natl Acad $S c i$ USA 1990;87:2951-4.

25 Petersen MB, Economou EP, Slaugenhaupt SA, Chakravarti $A$, Antonarakis SE. Linkage analysis of the human HMG14 gene on chromosome 21 using a GT dinucleotide repeat as polymorphic marker. Genomics 1990;7:136tide

26 Pinkel D, Straume T, Gray JW. Cytogenetic analysis using quantitative high-sensitivity, fluorescence hybridization. Proc Natl Acad Sci USA 1986;83:2934-8.

27 Koch J, Kølvraa S, Bolund L. An improved method for labelling of DNA probes by nicktranslation. Nucleic Acids Res 1986;14:7132.

28 Hansen MF, Cavenee WK. Retinoblastoma and the progression of tumor genetics. Trends Genet 1988;4:125-8. 